\title{
Clinical Study \\ Pregabalin Effect on Acute and Chronic Pain after Cardiac Surgery
}

\begin{abstract}
Aik Bouzia, ${ }^{1}$ Vassilios Tassoudis, ${ }^{2}$ Menelaos Karanikolas, ${ }^{3}$ George Vretzakis, ${ }^{2}$ Argyro Petsiti, ${ }^{2}$ Nikolaos Tsilimingas, ${ }^{4}$ and Elena Arnaoutoglou ${ }^{2}$
\end{abstract}

\author{
${ }^{1}$ Intensive Care Unit, Medical School of Larissa, University of Thessaly, Volos, Greece \\ ${ }^{2}$ Department of Anesthesiology, Medical School of Larissa, University of Thessaly, Volos, Greece \\ ${ }^{3}$ Department of Anesthesiology, Washington University School of Medicine, St. Louis, MO, USA \\ ${ }^{4}$ Department of Cardiothoracic Surgery, Medical School of Larissa, University of Thessaly, Volos, Greece
}

Correspondence should be addressed to Menelaos Karanikolas; kmenelaos@yahoo.com

Received 13 December 2016; Revised 20 March 2017; Accepted 5 April 2017; Published 30 April 2017

Academic Editor: Ronald G. Pearl

Copyright (C) 2017 Aik Bouzia et al. This is an open access article distributed under the Creative Commons Attribution License, which permits unrestricted use, distribution, and reproduction in any medium, provided the original work is properly cited.

\begin{abstract}
Introduction. Pain after cardiac surgery affects long-term patient wellness. This study investigated the effect of preoperative pregabalin on acute and chronic pain after elective cardiac surgery with median sternotomy. Methods. Prospective double blind study. 93 cardiac surgery patients were randomly assigned into three groups: Group 1 received placebo, Group 2 received oral pregabalin $75 \mathrm{mg}$, and Group 3 received oral pregabalin $150 \mathrm{mg}$. Data were collected 8 hours, 24 hours, and 3 months postoperatively. Results. Patients receiving pregabalin required fewer morphine boluses (10 in controls versus 6 in Group 1 versus 4 in Group 2, $p=0.000$ ) and had lower pain scores at 8 hours ( 4 versus 3 versus $3, p=0.001$ ) and 3 months ( 3 versus 2 versus $2, p=0.000$ ) and lower morphine consumption at 8 hours (14 versus 13 versus $12 \mathrm{mg}, p=0.000)$ and 24 hours (19.5 versus 16 versus $15 \mathrm{mg}$, $p=0.000$ ). Percentage of patients with sleep disturbances or requiring analgesics was lower in the pregabalin group and even lower with higher pregabalin dose (16/31 versus $5 / 31$ versus $3 / 31, p=0.000$, and $26 / 31$ versus $16 / 31$ versus $10 / 31, p=0.000$, resp.) 3 months after surgery. Conclusion. Preoperative oral pregabalin 75 or $150 \mathrm{mg}$ reduces postoperative morphine requirements and acute and chronic pain after cardiac surgery.
\end{abstract}

\section{Introduction}

Acute postsurgical pain and the possible progression to chronic postsurgical pain (CPSP) can influence patients' immediate and late postoperative course after cardiac surgery [1]. Sources of pain after cardiac surgery with median sternotomy include the sternotomy incision, chest tubes, the pericardial drainage, the site of saphenous vein, or radial artery harvesting $[2,3]$. The pain is described as chest discomfort of noncardiac origin in up to $65 \%$ of cases and can coexist with pain in the upper extremities, neck, head, and midback area [4]. Acute postoperative pain after coronary artery bypass grafting (CABG) surgery adversely affects pulmonary function in the first two postoperative days and can potentially delay extubation and prolong hospital stay [5, 6], thereby adversely affecting patient recovery. Furthermore, acute pain can progress to chronic pain with hypoesthesia and allodynia along the surgical incision. 56\% of patients describe sternotomy pain as pain of medium intensity that affects daily activities, while $38 \%$ of patients report unbearable pain. Chronic pain after cardiac surgery can adversely influence quality of life even a year after surgery [7-10], with one-third of patients with chronic pain reporting sleep disturbances. In patients over 60 years of age, pain has been recorded even 28 months after surgery with reported frequency as high as $40 \%$. Studies in female patients showed that pain persisted over a year with a significant percentage requiring treatment by a physician or physiotherapist [10].

Approaches used in an attempt to reduce pain after cardiac surgery include local anesthetic infiltration of the sternotomy and chest tube insertion sites [11], parasternal block [12], thoracic epidural analgesia [13, 14], and analgesic 
medications, including intravenous opioids $[15,16]$, intrathecal opioids [17], and paracetamol, diclofenac, and gabapentin by different routes [18-20].

Pregabalin (Lyrica, Pfizer, Inc.) is a beta-isobutyl of GABA with chemical similarity to gabapentin [21]. Pregabalin binds to the alpha-2-delta subgroup of calcium channels, thereby reducing excitatory neurotransmitter release and preventing hyperalgesia and central sensitization [22]. Compared to gabapentin, pregabalin seems to have more potent analgesic effects with fewer adverse effects [21]. Pregabalin is used as anticonvulsant but has also been used as analgesic for neuropathic pain and, lately, for postoperative pain, in an attempt to reduce opioid consumption and prevent progression to chronic pain [23-25].

Pregabalin may have a role in controlling acute postoperative pain and published evidence suggests that it may be effective in prevention of chronic postsurgical pain (CPSP) $[24,26,27]$. In addition, there is evidence that pregabalin confers an opioid-sparing effect in patients undergoing cardiac surgery [28]. Therefore, it is plausible that pregabalin could reduce acute pain and opioid requirements after cardiac surgery and may help prevent the transition from acute to chronic pain after cardiac surgery. This study was conducted to investigate the impact of a single preoperative oral pregabalin dose on morphine consumption in the immediate postoperative period and on the transition from acute to chronic pain in patients undergoing elective cardiac surgery with median sternotomy.

\section{Methods}

After approval by the Ethics Committee, 93 patients scheduled for cardiac surgery in a tertiary care University Hospital enrolled in this double blind, placebo controlled, randomized study. The study protocol was in agreement with the Helsinki Declaration on patient safety during anesthesiology research [29] and was registered in the "ClinicalTrials.gov" clinical trial registration website (ClinicalTrials.gov identifier: NCT01701921).

Inclusion criteria were elective primary cardiac surgery with median sternotomy and extracorporeal circulation, ages 18-85 years, and written informed consent for participation in the study. Exclusion criteria included previous cardiac or thoracic surgery, allergy to pregabalin, previous use of gabapentin or pregabalin, chronic pain, known diagnosis of depression or other major psychiatric diseases, cognitive impairment or inability to cooperate with the study, renal insufficiency, and history of substance abuse. Patient demographic and clinical data were stored in a secure, encrypted electronic database. Based on a computer custom number generator, patients were randomly assigned to one of three groups: Group 1 = control (patients received a placebo capsule), Group 2 = low dose pregabalin (patients received a capsule containing $75 \mathrm{mg}$ of pregabalin) (Lyrica, Pfizer Ltd.), and patients in Group 3 = high dose pregabalin received a capsule containing $150 \mathrm{mg}$ of pregabalin. The study drug (pregabalin or placebo) was given to patients by a research coordinator and was documented in the medical record as "study drug."
All anesthesia personnel taking care of patients in the operating room were blinded to group assignment.

After introduction of a large intravenous catheter in the upper extremity and placement of standard monitoring (ECG, noninvasive blood pressure, pulse oximetry, and capnography) the radial artery was cannulated under local anesthesia. Then, a bispectral index (BIS) sensor was placed, general anesthesia was induced using etomidate $0.3 \mathrm{mg} / \mathrm{kg}$ and fentanyl $30 \mathrm{mcg} / \mathrm{kg}$, and intubation was facilitated with vecuronium $0.1 \mathrm{mg} / \mathrm{kg}$. Anesthesia was initially maintained using sevoflurane at 1-1.2 age-adjusted MAC until insertion of a cordis in the right internal jugular vein and placement of a continuous cardiac output/mixed venous oximetry pulmonary artery catheter. Then, after central line placement was completed, anesthesia was maintained using propofol $100-200 \mu \mathrm{g} / \mathrm{kg} / \mathrm{minute}$ and remifentanil infusion $0.1-0.2 \mu \mathrm{g} /$ $\mathrm{kg} / \mathrm{min}$, titrated to maintain BIS values in the 35 to 50 range, and sevoflurane was discontinued. All operations were performed by the same surgeon, through midline sternotomy, using cardiopulmonary bypass with a roller pump and membrane oxygenator; pump flow rate was approximately $2.5 \mathrm{~L} /$ $\mathrm{min} / \mathrm{m}^{2}$ in order to maintain perfusion pressure around 50 $\mathrm{mm} \mathrm{Hg}$. During cardiopulmonary bypass, core temperature was normal for coronary artery bypass graft (CABG) procedures, while mild hypothermia was applied for valve replacement operations. At the end of surgery, patients remained intubated and were transported to the ICU under continuing propofol and remifentanil infusion for sedation. Criteria for weaning from the ventilator and extubation were hemodynamic stability, absence of excessive chest tube drainage or arrhythmias, normothermia, adequate urine output, spontaneous ventilatory frequency $<20$ breaths $/ \mathrm{min}$, and a cooperative patient that could respond to basic commands. After extubation, when patient condition was satisfactory, patients received a single $5 \mathrm{mg}$ dose of intravenous morphine and patient control analgesia (PCA) was started with a programmable PCA pump (Gemstar, Abbott) using the following settings: basal morphine infusion $0.5 \mathrm{mg} /$ hour, bolus dose 0.5 $\mathrm{mg}$, and lockout time 30 minutes. Analgesia was supplemented with IV paracetamol $1 \mathrm{gm}$ every 8 hours for the first 24 hours after surgery. In addition, if the analgesic regimen was not sufficient despite maximum PCA use, we advised ICU clinicians to provide additional (rescue) analgesia with IV morphine as deemed necessary. The goal was to keep pain scores measured by Verbal Rating Scale (VRS) $(0=$ no pain to $10=$ unbearable pain) at rest $\leq 4$ for every patient. An anesthesiologist blinded to group assignment visited the patients in 8 hours and 24 hours after extubation, recorded VRS after a deep breath, documented the presence and severity of nausea and vomiting, and collected data on PCA use and morphine consumption from the Gemstar pump. In addition, a blinded searcher interviewed all patients by telephone 3 months after surgery, inquired about the impact of the operation on their lives, and asked specific questions about the presence and severity of pain after surgery. In cases where patients indicated they were still experiencing pain, the researcher also collected data about use of analgesics and the presence of sleep disorders. 
TABLE 1: Demographic and clinical patient characteristics. Data reported as mean \pm SD, median (range), or number of patients, as appropriate. Values were compared between groups using ANOVA, Kruskal-Wallis, or Chi-square tests as appropriate. $p$ values for significance were adjusted to $0.05 / 20=0.0025$ using Bonferroni correction for multiple comparisons.

\begin{tabular}{|c|c|c|c|c|}
\hline Variable & $\begin{array}{c}\text { Group } 1 \text { (control) } \\
\qquad N=31\end{array}$ & $\begin{array}{c}\text { Group } 2 \text { (pregabalin } 75 \mathrm{mg} \text { ) } \\
\qquad N=31\end{array}$ & $\begin{array}{c}\text { Group } 3 \text { (pregabalin } 150 \mathrm{mg} \text { ) } \\
\qquad N=31\end{array}$ & $p$ \\
\hline Age & $66.1 \pm 10.2$ & $67.4 \pm 7.8$ & $67.8 \pm 6.8$ & 0.705 \\
\hline $\operatorname{Sex}(M / F)$ & $21 / 10$ & $19 / 12$ & $17 / 14$ & 0.58 \\
\hline Weight & $82.2 \pm 14.5$ & $80.3 \pm 12.0$ & $79.6 \pm 8.9$ & 0.665 \\
\hline Height & $1.70 \pm 0.07$ & $1.68 \pm 0.07$ & $1.67 \pm 0.07$ & 0.342 \\
\hline BMI & $28.6 \pm 4.8$ & $28.4 \pm 3.9$ & $28.6 \pm 3.2$ & 0.973 \\
\hline Operation time & $243.2 \pm 29.6$ & $244.7 \pm 24.2$ & $221.9 \pm 27.4$ & 0.002 \\
\hline ASA status & $3(3,4)$ & $3(3,4)$ & $3(3,4)$ & 0.109 \\
\hline Hypertension & 18 & 23 & 28 & 0.015 \\
\hline Diabetes & 15 & 13 & 9 & 0.285 \\
\hline Hyperlipidemia & 21 & 19 & 22 & 0.713 \\
\hline Arrhythmias & 3 & 7 & 0 & 0.016 \\
\hline Thyroid disease & 0 & 3 & 1 & 0.161 \\
\hline Statins & 24 & 23 & 24 & 0.942 \\
\hline B-blockers & 25 & 16 & 20 & 0.030 \\
\hline Ca channel antagonists & 8 & 8 & 8 & 1.000 \\
\hline Diuretics & 9 & 10 & 11 & 0.863 \\
\hline ACE inhibitors & 10 & 10 & 7 & 0.625 \\
\hline ARBs & 10 & 8 & 9 & 0.855 \\
\hline Nitrates & 6 & 4 & 3 & 0.535 \\
\hline Antiplatelet agents & 12 & 19 & 16 & 0.204 \\
\hline
\end{tabular}

$\mathrm{ACE}=$ angiotensin-converting enzyme, $\mathrm{ANOVA}=$ Analysis of Variance, $\mathrm{ARBs}=$ Angiotensin Receptor Blockers, ASA = American Society of Anesthesiologists, AVR = Aortic Valve Replacement, CABG = Coronary Artery Bypass Graft, MVR = Mitral Valve Replacement.

2.1. Statistical Analysis. Sample size calculation was based on assumptions supported by the findings of an earlier pilot, unpublished study, as follows: primary outcome is morphine consumption at 24 hours, morphine consumption at 24 hours is mean $=20 \mathrm{mg}$, with $\mathrm{SD}=5 \mathrm{mg}$, and a change of morphine use by $4 \mathrm{mg}$ is a clinically meaningful change. Using these assumptions, the required sample size to have alpha error = 0.05 and power $=0.8$ is 26 patients per group. Based on this calculation, we decided to increase sample size by $20 \%$ to 32 patients per group, in order to reduce the risk of not having adequate sample size due to patient attrition, missing data, errors in patient allocation, or other study shortcomings.

Normality of continuous data was assessed with the Kolmogorov-Smirnov and Shapiro-Wilk tests, and findings regarding normality were visually validated with normal Qto-Q plots and detrended normal Q-to-Q plots. Based on this analysis, data on age, weight, height, BMI, and operation times were analyzed using ANOVA, whereas all other continuous variables were analyzed using the nonparametric Kruskal-Wallis test. When ANOVA or Kruskal-Wallis test showed a significant difference between groups for a particular variable, Student's $t$-test or the Mann-Whitney test was used for post hoc testing, to ascertain which pair of groups had significantly different values.

Frequencies for binary (yes/no) variables such as hypertension, arrhythmia, vomiting in the first 24 hours, use of analgesics, or sleep disturbances after 3 months were compared between groups using the Chi-square test. Because of the high number of comparisons during data analysis, we adjusted $p$ values for significance using the Bonferroni method, by dividing 0.05 by the number of comparisons $[30,31]$. Therefore, $p$ value for significance was adjusted to $0.05 / 20=0.0025$ for the demographic and comorbidity variables presented in Table 1 and to $0.05 / 12=0.0042$ for outcome variables presented in Table 2 , so that only $p$ values $<0.0025$ or 0.0042 , respectively, were considered significant. The Statistical Package for Social Sciences (IBM, SPSS statistics, version 22) was used for data analysis.

\section{Results}

Of 108 eligible patients, 101 patients enrolled in the study, and 93 patients completed the study (36 women, 57 men) as shown in the CONSORT diagram in Figure 1. Demographic and clinical patient data are summarized in Table 1.

Patient age was (mean \pm SD) $67.1 \pm 8.3$ years, weight was $80.7 \pm 11.9 \mathrm{Kg}$, height was $168 \pm 7 \mathrm{~cm}$, and BMI was $28.5 \pm 3$, while median ASA physical status was 3 (range 3-4). Frequency of comorbidities and medication use initially seemed significantly different between groups for several variables. However, after adjusting the significance level to $0.05 / 20$, therefore $p<0.0025$ (Bonferroni correction), 
TABLE 2: Postoperative analgesic use and pain intensity. Data reported as median (minimum, maximum). $p$ values for comparisons between study groups were calculated using the Kruskal-Wallis or Chi-square tests as appropriate. $p$ values for significance were adjusted to $0.05 / 12=$ 0.0042 using Bonferroni correction for multiple comparisons.

\begin{tabular}{|c|c|c|c|c|}
\hline Variable & $\begin{array}{c}\text { Group } 1 \text { (control) } \\
\qquad N=31\end{array}$ & $\begin{array}{c}\text { Group } 2 \text { (pregabalin } 75 \mathrm{mg} \text { ) } \\
\qquad N=31\end{array}$ & $\begin{array}{l}\text { Group } 3 \text { (pregabalin } 150 \mathrm{mg} \text { ) } \\
\qquad N=31\end{array}$ & $p$ \\
\hline Intraoperative fentanyl (mcg) & $200(150,350)$ & $200(100,350)$ & $200(50,300)$ & 0.689 \\
\hline Intraoperative remifentanil (mcg) & $420(380,600)$ & $420(360,480)$ & $400(360,500)$ & 0.168 \\
\hline Boluses requested & $12(4,74)$ & $6(0,27)$ & $4(1,26)$ & 0.000 \\
\hline Boluses given & $10(4,28)$ & $6(0,14)$ & $4(1,13)$ & 0.000 \\
\hline VRS at 8 hours & $4(2,6)$ & $3(3,4)$ & $3(0,6)$ & 0.001 \\
\hline VRS at 24 hours & $1(0,5)$ & $1(0,4)$ & $0(0,3)$ & 0.007 \\
\hline VRS at 3 months & $3(2,5)$ & $2(1,3)$ & $2(1,3)$ & 0.000 \\
\hline Morphine use in first 8 hours (mg) & $14(12,17)$ & $13(11,16)$ & $12(11,14)$ & 0.000 \\
\hline Morphine use in first 24 hours (mg) & $19.5(16,30)$ & $16(14,22)$ & $15(12.5,18)$ & 0.000 \\
\hline Vomiting at 24 hours & 6 & 4 & 3 & 0.535 \\
\hline Use of analgesics at 3 months & 26 & 16 & 10 & 0.000 \\
\hline Sleep disturbance at 3 months & 16 & 5 & 3 & 0.000 \\
\hline
\end{tabular}

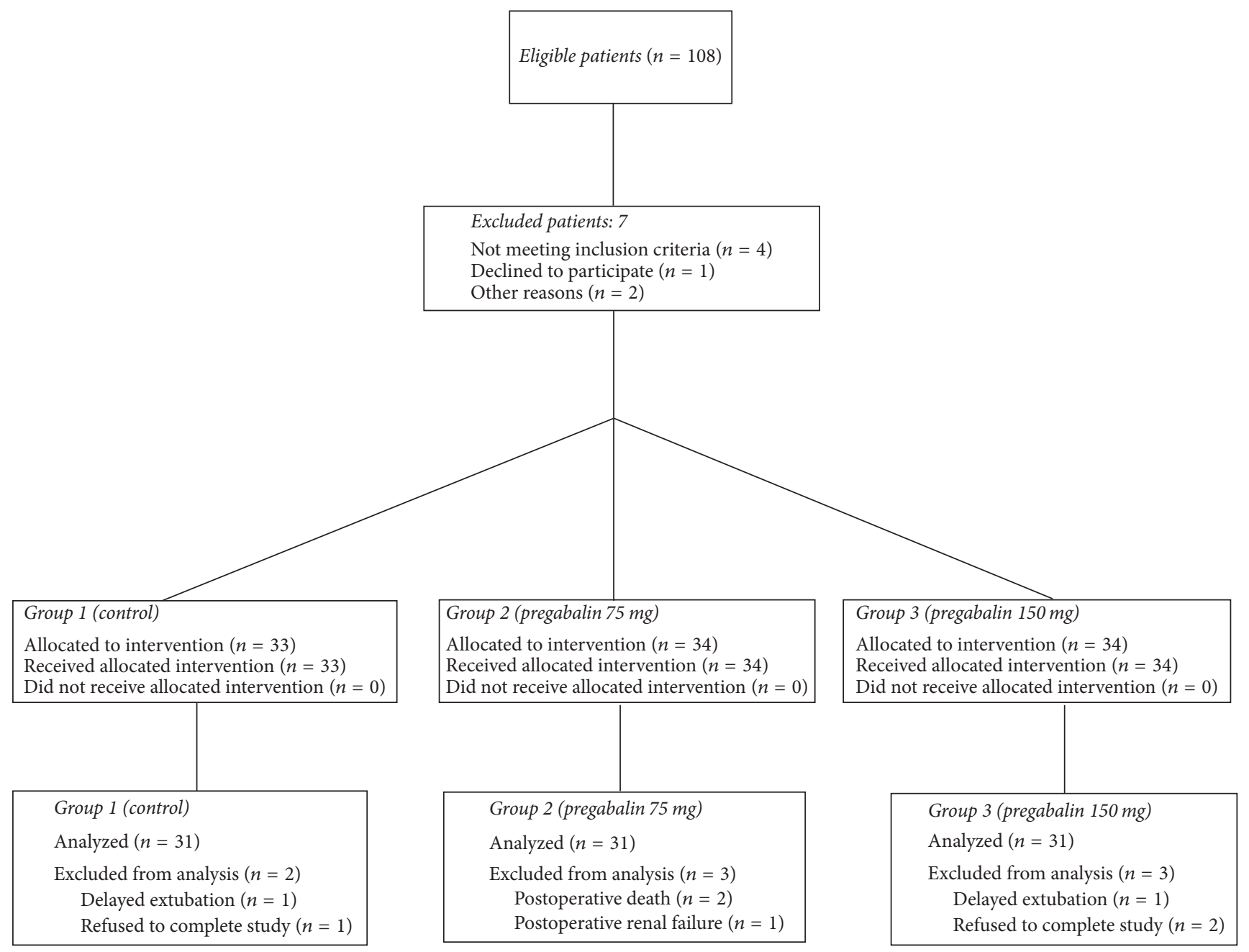

FIGURE 1: CONSORT diagram showing the progression of patients from eligibility to enrollment to completion of the study. 
TABLE 3: Post hoc comparisons between groups using the Mann-Whitney test.

\begin{tabular}{lcccccccc}
\hline Groups compared & $\begin{array}{c}\text { Boluses } \\
\text { requested }\end{array}$ & $\begin{array}{c}\text { Boluses } \\
\text { given }\end{array}$ & $\begin{array}{c}\text { VRS 8 } \\
\text { hours }\end{array}$ & $\begin{array}{c}\text { VRS 3 } \\
\text { months }\end{array}$ & $\begin{array}{c}\text { Morphine } \\
\text { consumption } \\
8 \text { hours }\end{array}$ & $\begin{array}{c}\text { Morphine } \\
\text { consumption } \\
\text { 24 hours }\end{array}$ & $\begin{array}{c}\text { Analgesics 3 } \\
\text { months }\end{array}$ & $\begin{array}{c}\text { Sleep } \\
\text { disturbance 3 } \\
\text { months }\end{array}$ \\
\hline $\begin{array}{l}\text { 1 versus 2 (control versus } \\
\text { pregabalin 75 mg) }\end{array}$ & 0.000 & 0.000 & 0.002 & 0.000 & 0.01 & 0.000 & 0.007 & 0.003 \\
$\begin{array}{l}1 \text { versus 3 (control versus } \\
\text { pregabalin 150 mg) }\end{array}$ & 0.000 & 0.000 & 0.002 & 0.000 & 0.000 & 0.000 & 0.000 & 0.000 \\
$\begin{array}{l}2 \text { versus 3 (pregabalin 75 mg } \\
\text { versus pregabalin 150 mg) }\end{array}$ & 0.003 & 0.004 & 0.465 & 0.985 & 0.1 & 0.005 & 0.126 & 0.452 \\
\hline
\end{tabular}

TABLE 4: Fentanyl and morphine consumption and pain scores in patients who did versus patients who did not vomit. Data are presented as median (Min, Max). Groups were compared using the Mann-Whitney test.

\begin{tabular}{lccc}
\hline Vomiting & Yes $(n=13)$ & No $(n=80)$ & $p$ \\
\hline Fentanyl & $250(150,350)$ & $200(50,350)$ & 0.047 \\
Morphine use in first 8 hours & $14.0(13.0,16.0)$ & $13.0(11.0,17.0)$ & 0.001 \\
Morphine use in first 24 hours & $18.5(15.0,30.0)$ & $16.5(12.5,27.5)$ & 0.003 \\
VRS at 8 hours & $4(4,6)$ & $3(0,6)$ & 0.007 \\
VRS at 24 hours & $2(0,5)$ & $1(0,4)$ & 0.04 \\
VRS at 3 months & $3(2,5)$ & $2(1,4)$ & 0.005 \\
\hline
\end{tabular}

only duration of surgery was significantly different between groups, being significantly shorter in Group $3(p=0.002)$.

Data on analgesic use, adverse effects, acute postoperative pain, chronic pain, and sleep disturbances are presented in Table 2. Morphine consumption recorded from the PCA as presented in the table did not include an initial $5 \mathrm{mg}$ dose given to all patients before extubation. $p$ values for significance were adjusted to 0.0042 using the Bonferroni correction, as described in Methods in order to avoid false positive findings due to multiple comparisons. Differences between groups remained significant for most variables of interest even after adjusting $p$ value for significance to 0.0042: the number of morphine dose requests and doses given, total morphine use in the first 8 hours and the first 24 hours, pain scores at 8 hours and at 3 months, and the frequency of sleep disturbances at 3 months were significantly different between the three groups.

When the overall testing showed a significant difference between groups for a variable of interest, as presented in Table 2, we then conducted post hoc testing using the Mann-Whitney test, in order to see which pair of groups was different with regard to each variable. Results of post hoc comparisons using the Mann-Whitney test are presented in Table 3. This table only includes variables where the overall test indicated the presence of a significant difference between groups.

With regard to vomiting, which can be very distressing for patients, there was no significant difference between groups. However, further analysis showed that morphine consumption at 8 and at 24 hours and pain scores at 8 hours and at 3 months were significantly higher in patients who experienced vomiting compared to those who did not (Table 4).

\section{Discussion}

Our results suggest that a single dose of pregabalin can result in a small but potentially beneficial reduction of acute postoperative pain and opioid consumption in the immediate postoperative period and may also reduce chronic poststernotomy pain in patients undergoing elective cardiac surgery with median sternotomy and use of cardiopulmonary bypass. Pregabalin, when added to the analgesic effect of morphine results in lower analgesia needs in the elderly, thereby leading to less severe acute postoperative pain and may potentially halt the progression to chronic pain, in accordance with the concept of "preventive analgesia" [32, 33].

Pregabalin has been used in different types of surgery and published clinical studies suggest that chronic pain may be reduced in patients who receive perioperative pregabalin $[24,26]$, but data on the role of pregabalin in cardiac surgery are limited: Ziyaeifard et al. [34] reported that a single preoperative dose of pregabalin $150 \mathrm{mg}$ significantly reduced pain but had no effect on morphine consumption after elective CABG surgery, whereas Joshi and Jagadeesh reported that one pregabalin dose before and one daily pregabalin dose for two days after surgery reduced pain scores and tramadol consumption for 36 hours but had no effect on chronic postoperative pain in patients undergoing off-pump coronary artery bypass (OPCAB) surgery [35]. However, a prospective clinical trial by Pesonen et al. showed that although perioperative pregabalin significantly reduced perioperative oxycodone consumption and the incidence of confusion, it increased the time to extubation in elderly patients undergoing cardiac surgery [28].

Considering the age of our patients (mean age $>65$ years, which is the official retirement age in many Western societies) 
we chose to only use a single dose of pregabalin $(75 \mathrm{mg}$ or $150 \mathrm{mg}$ ) in our study, in an attempt to minimize adverse effects in these elderly patients. Because the risk of confusion is high after cardiac surgery with cardiopulmonary bypass due to various mechanisms, including hypoperfusion, microemboli, and fast rewarming [36], we chose not to measure confusion, as we would not be able to attribute it to a specific factor. Instead, we used "number of bolus doses requested" as measure of patient agitation due to pain and "number of bolus doses given" as measure of patient analgesic needs and found that these variables differed significantly between the three groups. Although there is no obvious explanation for the observed differences between groups, it is reasonable to hypothesize that pregabalin reduces patient agitation and morphine requirement, and this effect is more pronounced with higher dose. This hypothesis is consistent with our data regarding morphine consumption at 8 hours and 24 hours after extubation where there are significant differences between all pairs of pregabalin groups.

Analysis of VRS in the first 8 hours after extubation showed significant difference between Groups 1 and 2 and Groups 1 and 3. After 24 hours the difference between Groups 1 and 2 is not significant, possibly because any difference is masked by increased morphine dose, but there is still significant difference between Groups 1 and 3. No difference is detected between Groups 2 and 3 at 8 and 24 hours. The above findings imply that a higher pregabalin dose is associated with less severe pain.

However, it is important to note that because the observed "average" differences in pain scores between groups were small, the clinical significance of such differences can be questioned. Similarly, the observed benefit with regard to reduced morphine consumption in the pregabalin groups is also small. Yet, we believe that the observed benefits with regard to pain scores and with regard to morphine consumption are clinically meaningful: differences in "maximum" scores are larger ( 2 points in most cases), and maximum pain is important from the viewpoint of patient experience. Similarly, maximum doses of morphine are markedly lower in patients who received pregabalin, and this reduction could be beneficial with regard to patient recovery and rehabilitation. Furthermore, it would not be realistic to expect a big, dramatic benefit from a single intervention in a complex, multifactorial care process, such as in cardiac surgery. Given the complexity of these cases, we believe that even a small reduction in pain intensity is a meaningful improvement as we all try to improve care for these patients. Three months after surgery the pattern is almost the same with patients in Group 1 experiencing more severe postoperative pain, using more analgesics and experiencing more sleep disturbances compared to patients in the pregabalin groups.

Although the small number of patients is a significant limitation of our study, the observed small but significant findings and the fact that these findings are biologically plausible and consistent with principles currently accepted in the pain literature support the validity of our findings. The observed significant difference in duration of surgery between groups, though statistically significant, is, in our opinion, of no clinical consequence and is unlikely to influence postoperative pain or any other relevant outcomes.

In conclusion, our data suggest that a single preoperative dose of oral pregabalin has small but potentially significant opioid-sparing effect, resulting in small but measurable improvement of postoperative pain with reduced morphine consumption and lower pain scores in the immediate postoperative period. The observed benefits with regard to opioid use and pain scores are small but clinically relevant, in our opinion, and may result, possibly through blockade of central sensitization mechanisms, in reduced long-term pain, with lower pain scores and fewer sleep disturbances 3 months after elective cardiac surgery. We believe that these results are truly promising. However, because this is a small, single center study and the observed clinical benefits are small, additional large, well conducted studies in different patient populations are needed to critically evaluate the validity of our findings.

\section{Conflicts of Interest}

The authors declare that they have no conflicts of interest.

\section{References}

[1] E. Kalso, S. Mennander, T. Tasmuth, and E. Nilsson, "Chronic post-sternotomy pain," Acta Anaesthesiologica Scandinavica, vol. 45, no. 8, pp. 935-939, 2001.

[2] C. Carle, A. Ashworth, and A. Roscoe, "A survey of poststernotomy chronic pain following cardiac surgery," Anaesthesia, vol. 64, no. 12, pp. 1387-1387, 2009.

[3] J. Bruce, N. Drury, A. S. Poobalan, R. R. Jeffrey, W. C. S. Smith, and W. A. Chambers, "The prevalence of chronic chest and leg pain following cardiac surgery: a historical cohort study," Pain, vol. 104, no. 1-2, pp. 265-273, 2003.

[4] N. J. Van Leersum, R. L. Van Leersum, H. F. Verwey, and R. J. M. Klautz, "Pain symptoms accompanying chronic poststernotomy pain: a pilot study," Pain Medicine, vol. 11, no. 11, pp. 1628-1634, 2010.

[5] A. Ergün and M. Sirlak, "Pulmonary function test before and after operation of coronary artery by-pass surgery," Tüberküloz ve Toraks, vol. 51, no. 1, pp. 17-22, 2003.

[6] M. C. D. S. Baumgarten, G. K. Garcia, M. H. Frantzeski et al., "Pain and pulmonary function in patients submitted to heart surgery via sternotomy," Brazilian Journal of Cardiovascular Surgery, vol. 24, no. 4, pp. 497-505, 2009.

[7] E. Eisenberg, Y. Pultorak, D. Pud, and Y. Bar-El, "Prevalence and characteristics of post coronary artery bypass graft surgery pain (PCP)," Pain, vol. 92, no. 1-2, pp. 11-17, 2001.

[8] K. M. King, M. Parry, D. Southern, P. Faris, and R. T. Tsuyuki, "Women's Recovery from Sternotomy-Extension (WREST-E) study: examining long-term pain and discomfort following sternotomy and their predictors," Heart, vol. 94, no. 4, pp. 493497, 2008.

[9] F. S. Routledge, R. T. Tsuyuki, M. Hervas-Malo, P. LeBlanc, J. A. McFetridge-Durdle, and K. M. King, "The influence of coronary artery bypass graft harvest site on women's pain, functional status, and health services utilization throughout the first postoperative year: a longitudinal study," International Journal of Nursing Studies, vol. 46, no. 8, pp. 1054-1060, 2009. 
[10] J. Meyerson, S. Thelin, T. Gordh, and R. Karlsten, "The incidence of chronic post-sternotomy pain after cardiac surgery-a prospective study," Acta Anaesthesiologica Scandinavica, vol. 45, no. 8, pp. 940-944, 2001.

[11] S. Kocabas, D. Yedicocuklu, E. Yuksel, E. Uysallar, and F. Askar, "Infiltration of the sternotomy wound and the mediastinal tube sites with $0.25 \%$ levobupivacaine as adjunctive treatment for postoperative pain after cardiac surgery," European Journal of Anaesthesiology, vol. 25, no. 10, pp. 842-849, 2008.

[12] S. B. McDonald, E. Jacobsohn, D. J. Kopacz et al., "Parasternal block and local anesthetic infiltration with levobupivacaine after cardiac surgery with desflurane: the effect on postoperative pain, pulmonary function, and tracheal extubation times," Anesthesia and Analgesia, vol. 100, no. 1, pp. 25-32, 2005.

[13] M. Caputo, H. Alwair, C. A. Rogers et al., "Thoracic epidural anesthesia improves early outcomes in patients undergoing offpump coronary artery bypass surgery: a prospective, randomized, controlled trial," Anesthesiology, vol. 114, no. 2, pp. 380390, 2011.

[14] Y. Mehta, M. Vats, M. Sharma, R. Arora, and N. Trehan, "Thoracic epidural analgesia for off-pump coronary artery bypass surgery in patients with chronic obstructive pulmonary disease," Annals of Cardiac Anaesthesia, vol. 13, no. 3, pp. 224-230, 2010.

[15] S. Baltali, A. Turkoz, N. Bozdogan et al., "The efficacy of intravenous patient-controlled remifentanil versus morphine anesthesia after coronary artery surgery," Journal of Cardiothoracic and Vascular Anesthesia, vol. 23, no. 2, pp. 170-174, 2009.

[16] A. Gurbet, S. Goren, S. Sahin, N. Uckunkaya, and G. Korfali, "Comparison of analgesic effects of morphine, fentanyl, and remifentanil with intravenous patient-controlled analgesia after cardiac surgery," Journal of Cardiothoracic and Vascular Anesthesia, vol. 18, no. 6, pp. 755-758, 2004.

[17] I. Bowler, G. Djaiani, R. Abel, S. Pugh, J. Dunne, and J. Hall, "A combination of intrathecal morphine and remifentanil anesthesia for fast-track cardiac anesthesia and surgery," Journal of Cardiothoracic and Vascular Anesthesia, vol. 16, no. 6, pp. 709$714,2002$.

[18] M. K. Fayaz, R. J. Abel, S. C. Pugh, J. E. Hall, G. Djaiani, and J. S. Mecklenburgh, "Opioid-sparing effects of diclofenac and paracetamol lead to improved outcomes after cardiac surgery," Journal of Cardiothoracic and Vascular Anesthesia, vol. 18, no. 6, pp. 742-747, 2004.

[19] N. Dhawan, S. Das, U. Kiran, S. Chauhan, A. K. Bisoi, and N. Makhija, "Effect of rectal diclofenac in reducing postoperative pain and rescue analgesia requirement after cardiac surgery," Pain Practice, vol. 9, no. 5, pp. 385-393, 2009.

[20] A. Ucak, B. Onan, H. Sen, I. Selcuk, A. Turan, and A. T. Yilmaz, "The effects of gabapentin on acute and chronic postoperative pain after coronary artery bypass graft surgery," Journal of Cardiothoracic and Vascular Anesthesia, vol. 25, no. 5, pp. 824-829, 2011.

[21] "Pregabalin: new drug. Very similar to gabapentin," Prescrire International, vol. 14, no. 80, pp. 203-206, 2005.

[22] J. Zhang, K.-Y. Ho, and Y. Wang, "Efficacy of pregabalin in acute postoperative pain: a meta-analysis," British Journal of Anaesthesia, vol. 106, no. 4, pp. 454-462, 2011.

[23] M. Dauri, S. Faria, A. Gatti, L. Celidonio, R. Carpenedo, and A. F. Sabato, "Gabapentin and pregabalin for the acute post-operative pain management. A systematic-narrative review of the recent clinical evidences," Current Drug Targets, vol. 10, no. 8, pp. 716-733, 2009.
[24] A. Buvanendran, J. S. Kroin, C. J. Della Valle, M. Kari, M. Moric, and K. J. Tuman, "Perioperative oral pregabalin reduces chronic pain after total knee arthroplasty: a prospective, randomized, controlled trial," Anesthesia and Analgesia, vol. 110, no. 1, pp. 199-207, 2010.

[25] R. A. Moore, S. Straube, P. J. Wiffen, S. Derry, and H. J. McQuay, "Pregabalin for acute and chronic pain in adults," Cochrane Database of Systematic Reviews, no. 3, p. CD007076, 2009.

[26] S. M. Burke and G. D. Shorten, "Perioperative pregabalin improves pain and functional outcomes 3 months after lumbar discectomy," Anesthesia and Analgesia, vol. 110, no. 4, pp. 1180$1185,2010$.

[27] H. Clarke, R. P. Bonin, B. A. Orser, M. Englesakis, D. N. Wijeysundera, and J. Katz, "The prevention of chronic postsurgical pain using gabapentin and pregabalin: a combined systematic review and meta-analysis," Anesthesia and Analgesia, vol. 115, no. 2, pp. 428-442, 2012.

[28] A. Pesonen, R. Suojaranta-Ylinen, E. Hammarén et al., "Pregabalin has an opioid-sparing effect in elderly patients after cardiac surgery: a randomized placebo-controlled trial," British Journal of Anaesthesia, vol. 106, no. 6, pp. 873-881, 2011.

[29] J. Mellin-Olsen, S. Staender, D. K. Whitaker, and A. F. Smith, "The helsinki declaration on patient safety in anaesthesiology," European Journal of Anaesthesiology, vol. 27, no. 7, pp. 592-597, 2010.

[30] J. M. Bland and D. G. Altman, "Multiple significance tests: the Bonferroni method," British Medical Journal, vol. 310, no. 6973, p. 170, 1995.

[31] M. Aickin and H. Gensler, "Adjusting for multiple testing when reporting research results: the Bonferroni vs Holm methods," American Journal of Public Health, vol. 86, no. 5, pp. 726-728, 1996.

[32] H. Clarke, M. Poon, A. Weinrib, R. Katznelson, K. Wentlandt, and J. Katz, "Preventive analgesia and novel strategies for the prevention of chronic post-surgical pain," Drugs, vol. 75, no. 4, pp. 339-351, 2015.

[33] F. Cavaliere and G. Draisci, "Pre-emptive or preventive analgesia?” Minerva Anestesiologica, vol. 79, no. 5, pp. 482-484, 2013.

[34] M. Ziyaeifard, M. J. Mehrabanian, S. Z. Faritus et al., "Premedication with oral pregabalin for the prevention of acute postsurgical pain in coronary artery bypass surgery," Anesthesiology and Pain Medicine, vol. 5, no. 1, Article ID e24837, 2015.

[35] S. S. Joshi and A. M. Jagadeesh, "Efficacy of perioperative pregabalin in acute and chronic post-operative pain after off-pump coronary artery bypass surgery: a randomized, double-blind placebo controlled trial," Annals of Cardiac Anaesthesia, vol. 16, no. 3, pp. 180-185, 2013.

[36] J. R. Maldonado, "Pathoetiological model of delirium: a comprehensive understanding of the neurobiology of delirium and an evidence-based approach to prevention and treatment," Critical Care Clinics, vol. 24, no. 4, pp. 789-856, 2008. 


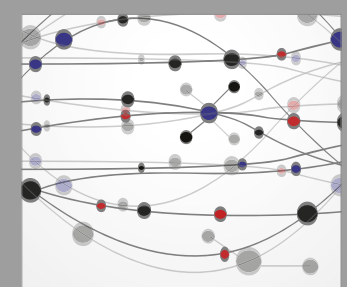

The Scientific World Journal
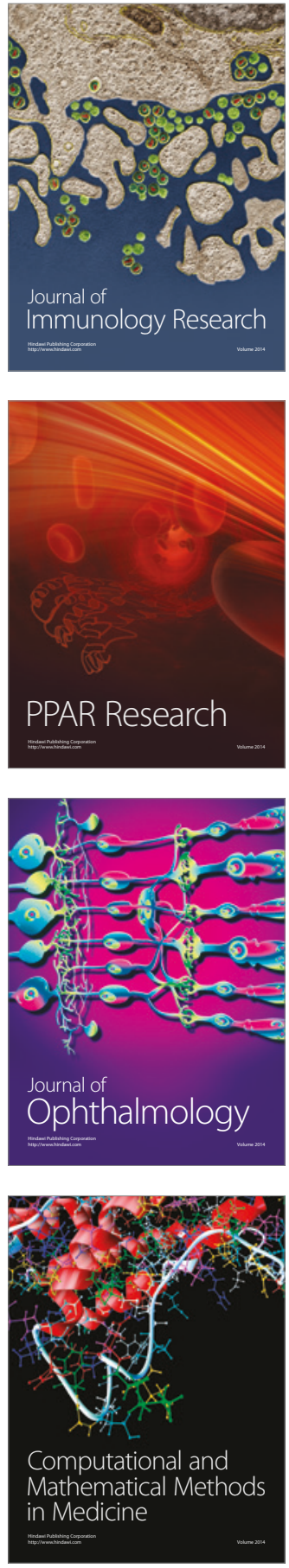

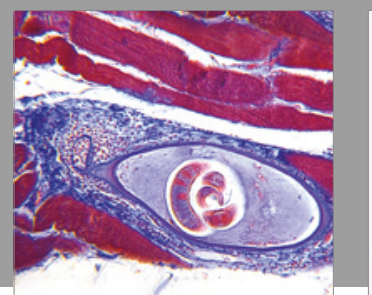

Gastroenterology Research and Practice
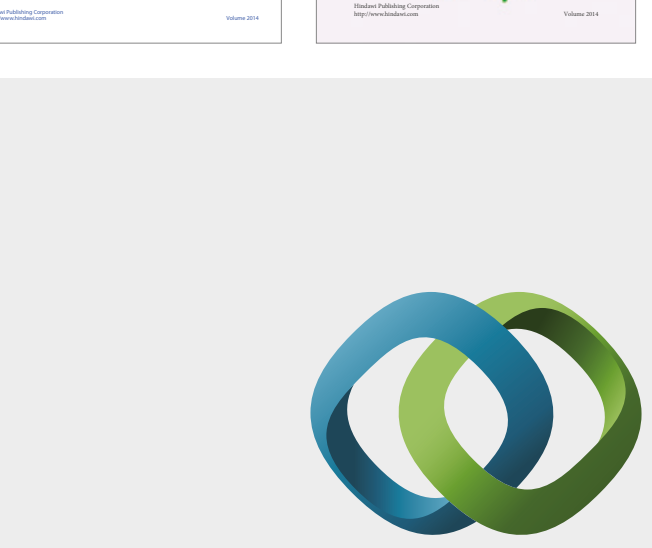

\section{Hindawi}

Submit your manuscripts at

https://www.hindawi.com
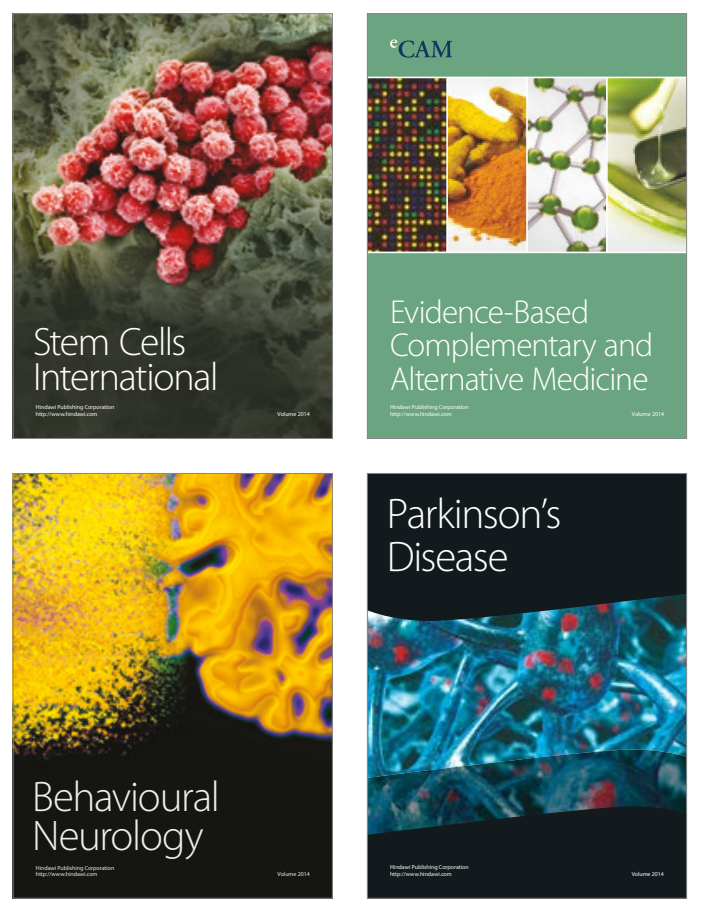
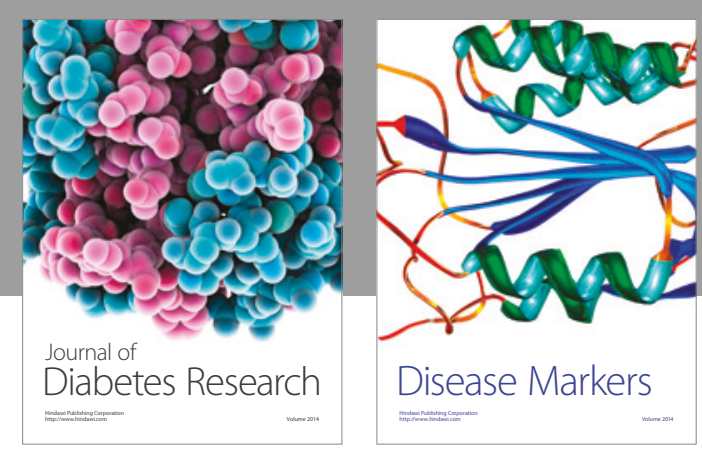

Disease Markers
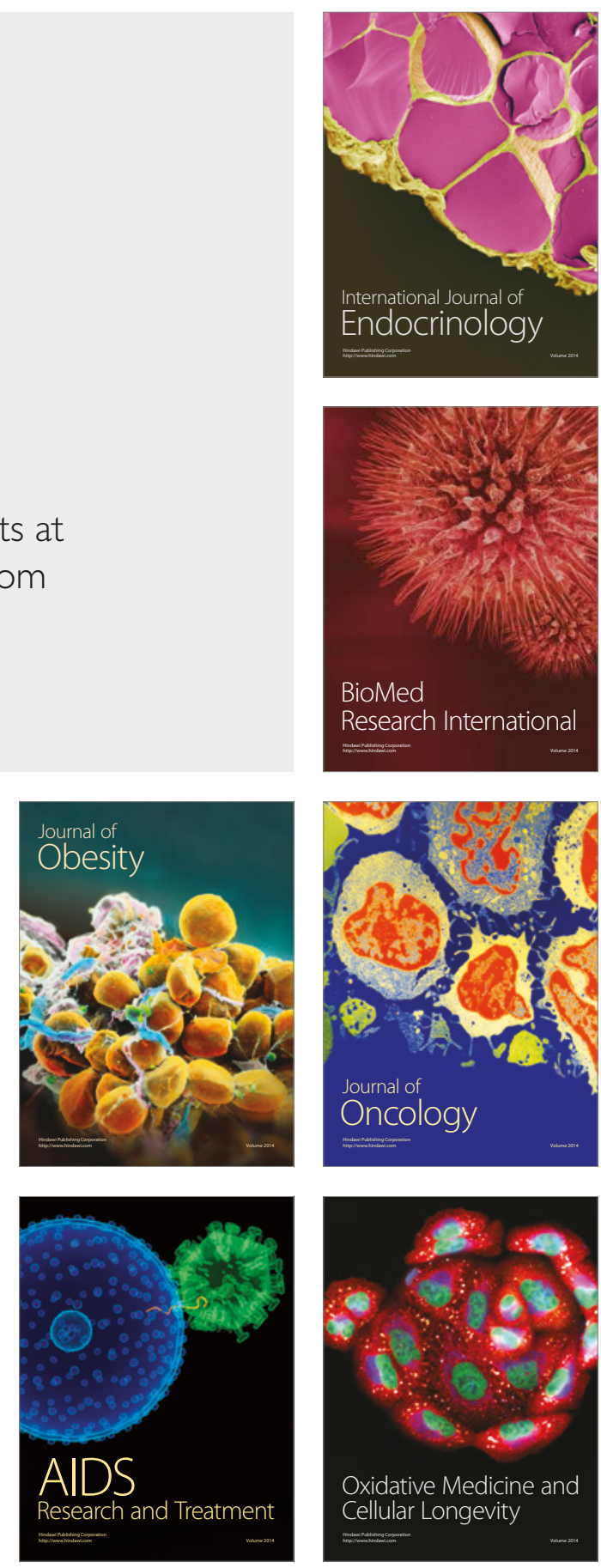